\title{
Selection of test bites records as part of a forensic bite mark analysis protocol
}

\author{
Fernando Rivera-Mendoza ${ }^{1,2}$, Stella Martín-de-las-Heras ${ }^{3}$, Pablo Navarro-Cáceres ${ }^{1,4}$ and Gabriel M. Fonseca ${ }^{1,2^{*}}$
}

\begin{abstract}
Background: Test bites have been recommended for collecting evidence of suspected dentition in bite mark analysis. However, these recommendations only suggest recording the interocclusal relationship without establishing number of records or characteristics to obtain proper records. This fails to consider not only the possibility that the recording material did not respond properly, but also that the evaluator has not detected simulations, habits or parafunctions that may affect the tests.

Methods: This research establishes differences between three wax test bites taken from the same subjects to determine if the first bite offered the best quality.

Results: No significant statistical association was found between the number of test bites recorded and respective quality. The interrater agreement produced almost perfect and substantial agreements.

Conclusion: There is no significant evidence that a first and only test bite is the highest quality test bite. Therefore, it is recommended to take more than a single bite test in controlled situations.
\end{abstract}

Keywords: Forensic science, Forensic odontology, Bite marks, Test bite records

\section{Background}

In bite mark cases, when authorities have identified a potential suspect, his or her dental records may provide the basis for comparison. It has been proposed that this comparison allows forensic odontologists to establish a correlation between the dental pattern of the suspect and that of the bite mark under analysis (Dailey 2011).

Sample bites (or test bites) have been suggested by the American Board of Forensic Odontology (2017) as part of the protocol for collecting evidence of suspected human dentition. These tests are tools that allow reproducing the biting edges of teeth in a position that simulates the bite mark pattern (Dailey 2011; American Board of Forensic Odontology 2017). Current standards have emphasized the type of materials used in this procedure, suggesting American Dental Association (ADA) certified materials (dental

\footnotetext{
* Correspondence: gabriel.fonseca@ufrontera.cl

${ }^{1}$ Programa de Magister en Odontología, Facultad de Odontología, Universidad de La Frontera, Temuco, Chile

${ }^{2}$ Forensic Dentistry Lab, Centro de Investigación en Odontología Legal y Forense (ClO), Facultad de Odontología, Universidad de La Frontera,

Francisco Salazar 01145, Building L, 4811230 Temuco, Chile

Full list of author information is available at the end of the article
}

waxes or silicone-based) or, with some limitations, expanded polystyrene foam (Styrofoam ${ }^{\circ}$ ) (American Board of Forensic Odontology 2017; Johnson 2011).

Great care must be taken that the opposing teeth do not come into contact with each other when the bite is recorded. This precaution helps avoid causing 3-D distortions in position, shape and alignment of the teeth (Dailey et al. 2013). In this context, ABFO suggests "recording the interocclusal relationship" without establishing preventive behavior, number of records or characteristics that reflect the complexity of the biting act of the suspect (American Board of Forensic Odontology 2017). According to technical logic, failure to establish a minimum number of records would make it acceptable to declare the first and only test "adequate". With this, the act of biting is limited to just "closing the teeth" and fails to consider not only the possibility that the recording material did not respond properly, but also that the evaluator has not detected simulations, habits or simple oral parafunctions that may compromise the quality of a sample.

Dental occlusion implies much more than the occlusal contact relationships of the dentitions and refers to a 
dynamic biomechanical musculoskeletal system (Jokstad 2012). There are millions of possible mandibular placements for occlusion's intercuspal position (considering the mandible's placement in all three dimensions of space) and the range of motion is influenced by a high number of factors. The neuromuscular system adapts easily to these factors determining an "engram" (the socalled "muscle memory"), which cannot be interpreted as a simple mechanical abstraction of motion in a single plane (Lerman 2011). The goal of this research was to establish if differences exist between three wax test bites (Webster's type 3 bites in which teeth biting right through or almost through the bitten material, typical of the bite found in cheese) (Webster 1982) taken from the same subjects, to corroborate if the first test bite was of superior quality, and to discuss the value of having more test bites to dynamically interpret the bite action. We also discuss some technical proposals to improve the test bites as well as the strategies of comparative analysis of them.

\section{Methods}

The present study was approved by the Ethical Scientific Committee of the Universidad de La Frontera (CEC) by act no. 079/2015. The CEC is an agency accredited by the Regional Ministerial Health Secretariat of Chile. The experiments carried out in this study were performed within the guidelines of the CEC for human studies and were carried out under the strict supervision of a registered dental practitioner (FRM). Also, all the subjects have been fully informed of the experimental procedure and consent was obtained prior to the experiment. Thirty asymptomatic subjects aged between 19 and 24 years, from the Facultad de Odontología of the Universidad de La Frontera (Temuco, Chile) volunteered for this study. Inclusionary criteria were to have natural healthy teeth (incisors, canines and first premolar) with a maxillary and mandibular alignment as close to ideal, scored using the Little's Irregularity Index (Little 1975) (from the ideal zero score to a maximum of $3 \mathrm{~mm}$ ). Exclusionary criteria included history of caries, supernumerary teeth, dental anomalies and direct or indirect dental rehabilitations in these teeth.

\section{Test bites}

All participants were invited to perform test bites on triangular blue wax wafers. These wafers were previously made Ad-hoc with Great Lakes Tapered Wafers 25/pkg. (Great Lakes Orthodontics Ltd., Tonawanda NY, USA) following the handling and storage guidelines provided by the manufacturer (Safety Data Sheet, available at www.greatlakesortho.com). This type of wax was chosen for its physical properties and biocompatibility (Table 1 ).
Table 1 Attributes of the Great Lakes Tapered Wafers 25/pkg. ${ }^{\oplus}$, according to Sections 3, 9, 11, and 12 of the Safety Data Sheet provided by the manufacturer (www.greatlakesortho.com)

\begin{tabular}{|c|c|}
\hline Product name & $\begin{array}{l}\text { Great Lakes Tapered Wafers 25/pkg. }{ }^{\oplus} \\
\text { (Wax, product number 260-032) }\end{array}$ \\
\hline Manufactured by & $\begin{array}{l}\text { Great Lakes Orthodontics Ltd., } \\
\text { Tonawanda NY, USA }\end{array}$ \\
\hline $\begin{array}{l}\text { Hazardous } \\
\text { components }\end{array}$ & $\begin{array}{l}\text { This material is not hazardous under the criteria } \\
\text { of the Federal OSHA Hazard Communication } \\
\text { Standard } 29 \text { CFR } 1910.1200\end{array}$ \\
\hline Form & Solid \\
\hline Color & Light Blue \\
\hline Odour & Mild odour \\
\hline Flash Point & $465^{\circ} \mathrm{F}\left(240^{\circ} \mathrm{C}\right)$ \\
\hline Boiling Point & $>450^{\circ} \mathrm{F}$ \\
\hline $\begin{array}{l}\text { Freezing/Melting } \\
\text { Point }\end{array}$ & $\begin{array}{l}\text { Softening Point by Ring and Ball Apparatus ASTM } \\
\text { E28-58 T: } 145^{\circ} \mathrm{F}\end{array}$ \\
\hline $\begin{array}{l}\text { Toxicological } \\
\text { information }\end{array}$ & $\begin{array}{l}\text { No evidence of adverse effects from available } \\
\text { information }\end{array}$ \\
\hline $\begin{array}{l}\text { Ecological information } \\
\text { (non mandatory) }\end{array}$ & $\begin{array}{l}\text { No evidence of adverse effects from available } \\
\text { information }\end{array}$ \\
\hline
\end{tabular}

The objective of this procedure was to obtain test bites that followed the criteria established by Webster (1982) for Type 3 bites (Table 2). This classification of bite marks in foodstuffs and inanimate objects has been recommended and, in fact, there seems to be no other attempt to standardize terminology for this type of evidence (Rivera-Mendoza et al. 2017). In order to obtain bite records that achieve these criteria, the guidelines were followed as established by Scott (2010) and Dawson (The Dawson Academy Blog 2008), and modified Ad-hoc for the present study (Fig. 1):

- The bite record must not cause any movement or displacement of teeth.

- Each subject was invited to perform three consecutive test bites on respective wafers, while

Table 2 Characteristics of Type 3 bites (adapted from Webster 1982)

- The teeth bite right through or almost through the bitten material.

- The bitten piece is removed by fracturing it from the main material.

- The bite shows a record of the outline of the labial aspect of the upper and lower incisor teeth.

- The tooth scrape marks penetrating the bitten material.

- Both labial outline marks and tooth scrape marks tend to record those elements of the teeth which are most prominent anteriorly.

- The widths of the tooth scrape marks are the horizontal width between

the most mesial and most distal parts of the tooth crown recorded.

- The body of the bite exhibits extensive scrape marks and may give an indication of the relative positions of the upper and lower incisor teeth. 


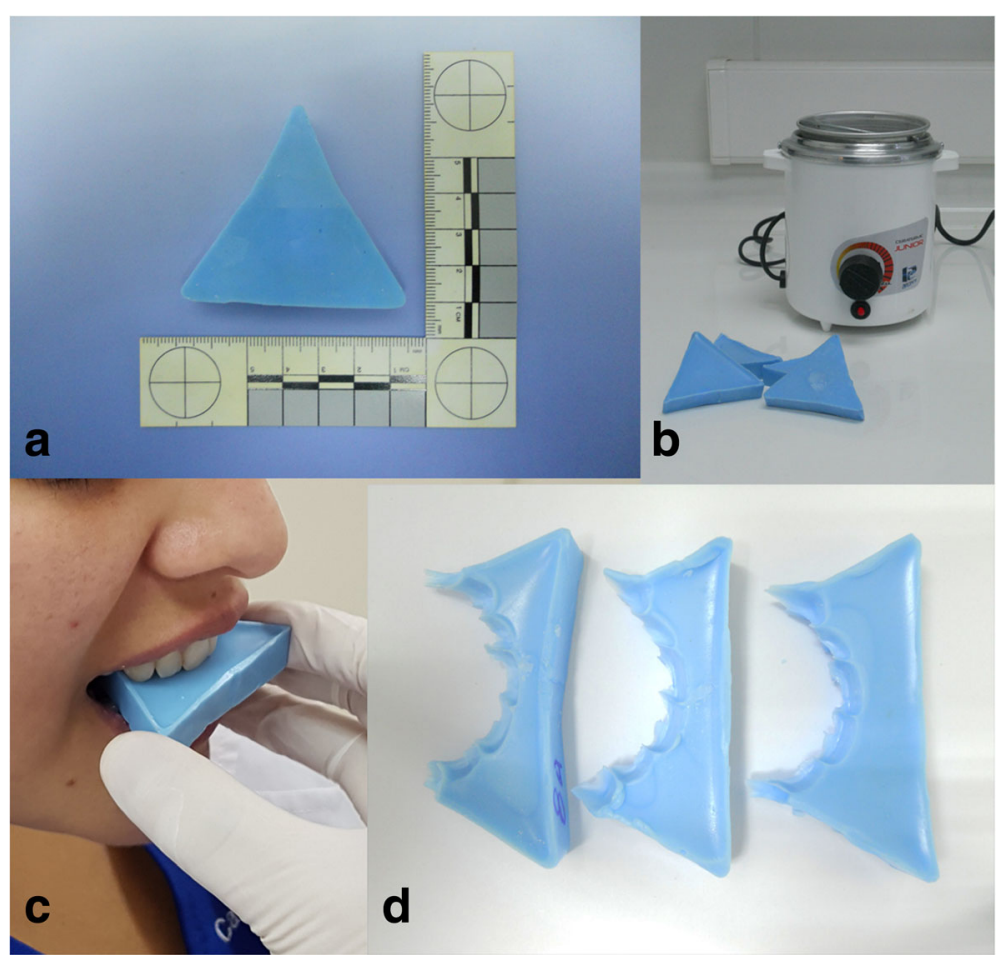

Fig. 1 Protocol for register the test bite records. a Triangular blue wax wafers made Ad-hoc with Great Lakes Tapered Wafers 25/pkg. . (Great Lakes Orthodontics Ltd., Tonawanda NY, USA). b Wax immersion in a thermostatically controlled water bath at $58^{\circ} \mathrm{C}$ for 60 s. c The subject was asked to perform three consecutive test bites on respective wafers ensuring a natural interocclusal record. $\mathbf{d}$ The wafers were removed in a single movement to avoid deformations and placed in cold water

ensuring accurate verification of the interocclusal record.

- In each case, the wax was softened to avoid distortion of the bite pattern. Since the wax is naturally hard, the wafers were immersed in a thermostatically controlled water bath at $58{ }^{\circ} \mathrm{C}$ for $60 \mathrm{~s}$. The wafers were inserted into the subject's mouth by placing them against the upper arch, pressing against the arch to ensure teeth indented the wax.

- In order to avoid modifying the subject's natural bite pattern, each subject was asked to try to contact all of his/her posterior teeth in the same biting act. The mandible was not manipulated in any way to allow the natural design of the engram during the arc of closure and to avoid overwhelming (Lerman 2011).

- The wafers were removed from the mouth while they were still warm and in a single movement to avoid deformations. Following the manufacturer's standards, the records were immediately placed in cold water to complete the hardening phase of the wax. Once performed, each of the records were checked, coded, anonymized and preserved following the manufacturer's recommendations to avoid environmental modifications.

\section{Analysis}

Following the tests, two trained observers (FRM, GMF) collaborated on evaluation and categorization of record quality. Both observers evaluated three records simultaneously and three independently for further consensus. For this, and as a previous calibration procedure, tests bites not included in the study sample were used. Following Sheasby \& MacDonald's criteria for "primary distortions" ("those occurred at the time of biting") (Sheasby and MacDonald 2001), and having discarded the test bites that did not respect the requirements established by Webster for type 3 (1982), the calibration allowed establishment of which were to be considered "patterns of slightest distortions" (Fig. 2):

- Wax runoff.

- Wax stretching.

- Presence of beveling on the biting edges of teeth.

- Presence of excesses or over-edges of wax.

- Incorrect definition of interproximal ridges.

- Incorrect intermaxillary relationship: lateral displacement.

- Incorrect intermaxillary relations: lack of deepening of the bite. 


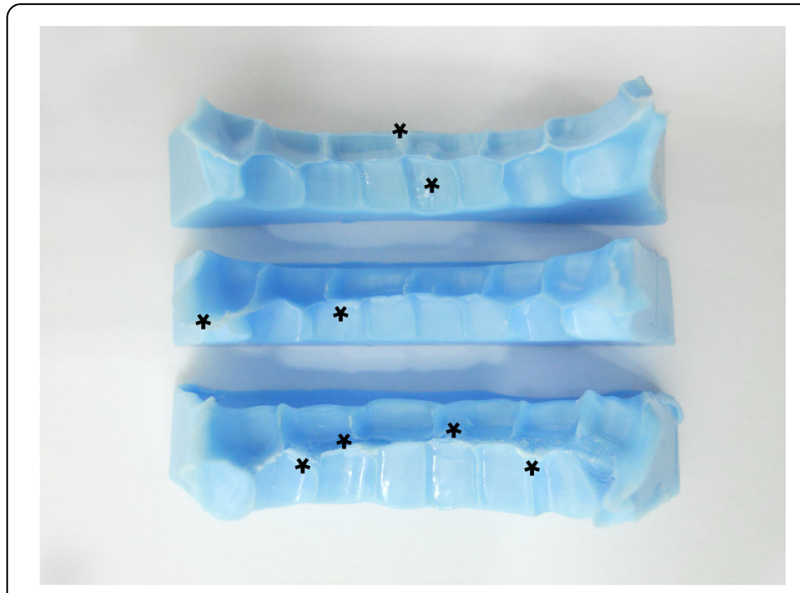

Fig. 2 First, second and third test bite record in the same subject. Asterisks point to different patterns of slightest distortions

These slightest distortion patterns were used to categorize each of the three records of each individual into three different categories of record quality, regardless of how many patterns they had: better, fair or worse. Seven days after all sampling test bites have been finalized, they were categorized according to this classification, and discrepancies were resolved by consensus. The records of the first sampling and the second sampling were compared according to a design of paired samples by individual. Pearson chi-square test was used to assess the association of the categories of record quality (CRQ) with the first, second or third test bite record. Likewise, interrater agreement was measured between the observers (Cohen's k) with results categorized as: poor agreement (below 0.0); slight agreement (0.00 to 0.20); fair agreement (0.21 to $0.40)$; moderate agreement (0.41 to 0.60$)$; substantial agreement (0.61 to 0.80); and almost perfect agreement (0.81 to 1.00$)$.

\section{Results}

No significant statistical association was found between the number of test bite record (first, second or third) and the three categories of record quality: CRQ better $(p=0.407)$, CRQ fair $(p=0.741)$ or CRQ worse ( $p=$ 0.741 ) (Table 3 ). The interrater agreement produced good results for all categories: CRQ better $(k=0.846$, almost perfect agreement), CRQ fair $(\mathrm{k}=0.700$, substantial agreement) and CRQ worse $(\mathrm{k}=0.797$, substantial agreement) (Table 4).

\section{Discussion}

Our results demonstrate that there is no evidence that a first (and only) test bite record is the best representation of the subject's bite action. This is not a minor issue, considering that no protocol suggests performing more
Table 3 Descriptive statistic showing the association between the number of test bite record and the categories of record quality. CRQ: Category of Record Quality

\begin{tabular}{|c|c|c|c|c|c|}
\hline & & Frequency & Percent & $\begin{array}{l}\text { Valid } \\
\text { Percent }\end{array}$ & $\begin{array}{l}\text { Cumulative } \\
\text { Percent }\end{array}$ \\
\hline \multicolumn{6}{|c|}{ CRQ: Better } \\
\hline \multirow[t]{4}{*}{ Valid } & 1st TEST BITE & 7 & 23.3 & 23.3 & 23.3 \\
\hline & 2nd TEST BITE & 10 & 33.3 & 33.3 & 56.7 \\
\hline & 3rd TEST BITE & 13 & 43.3 & 43.3 & 100.0 \\
\hline & Total & 30 & 100.0 & 100.0 & \\
\hline \multicolumn{6}{|c|}{$X^{2}=1.800^{a}$} \\
\hline \multicolumn{6}{|c|}{$p=.407$} \\
\hline \multicolumn{6}{|c|}{$C R Q:$ Fair } \\
\hline \multirow[t]{4}{*}{ Valid } & 1st TEST BITE & 11 & 36.7 & 36.7 & 36.7 \\
\hline & 2nd TEST BITE & 11 & 36.7 & 36.7 & 73.3 \\
\hline & 3rd TEST BITE & 8 & 26.7 & 26.7 & 100.0 \\
\hline & Total & 30 & 100.0 & 100.0 & \\
\hline \multicolumn{6}{|c|}{$X^{2}=.600^{a}$} \\
\hline \multicolumn{6}{|c|}{$p=.741$} \\
\hline \multicolumn{6}{|c|}{ CRQ: Worse } \\
\hline \multirow[t]{4}{*}{ Valid } & 1st TEST BITE & 12 & 40.0 & 40.0 & 40.0 \\
\hline & 2nd TEST BITE & 9 & 30.0 & 30.0 & 70.0 \\
\hline & 3rd TEST BITE & 9 & 30.0 & 30.0 & 100.0 \\
\hline & Total & 30 & 100.0 & 100.0 & \\
\hline \multicolumn{6}{|c|}{$X^{2}=.600^{a}$} \\
\hline \multicolumn{6}{|c|}{$p=.741$} \\
\hline
\end{tabular}
is 10.0

than one test bite record, which implies that slightest distortions may be overlooked if only one test bite record is considered. These slightest distortions do not necessarily represent errors in the recording procedures, but dynamic patterns that can manifest in varying degrees depending on multiple factors. In 2001, Sheasby \& MacDonald stated that since the nature of the contact between teeth and the bitten substrate can influence the resulting bite mark, it is logical to postulate that the dynamics of the biting action may produce distortions known as "primary distortions". According to these authors, these distortions are complex, unpredictable, and absolutely dependent and proportional to the degree of movement between the teeth and the bitten substrate (Sheasby and MacDonald 2001). It is extremely interesting to mention that although the authors report that each episode of contact is a unique dynamic event, and that the same dentition can produce bite marks with different forms of appearance (Sheasby and MacDonald 2001), we have not found a test bite protocol that explicitly considers performing more than one single record to control this phenomenon. The results of our research 
Table 4 Interrater Cohen's k scores. Contingency table and symmetric measures for the Categories of Record Quality (CRQ): Better, Fair and Worse. Obs.1 = Observer 1; Obs. 2 = Observer 2

\begin{tabular}{|c|c|c|c|c|c|}
\hline & & \multicolumn{3}{|l|}{ Obs. 2} & \multirow[t]{2}{*}{ Total (\%) } \\
\hline & & 1st TEST BITE (\%) & 2nd TEST BITE (\%) & 3rd TEST BITE (\%) & \\
\hline \multicolumn{6}{|c|}{ Contingency table for CRQ Better } \\
\hline \multirow[t]{3}{*}{ Obs.1 } & 1st TEST BITE (\%) & $6(20.0)$ & $1(3.3)$ & $0(0.0)$ & $7(23.3)$ \\
\hline & 2nd TEST BITE (\%) & $0(0.0)$ & $10(33.3)$ & $1(3.3)$ & $11(36.7)$ \\
\hline & 3rd TEST BITE (\%) & $1(3.3)$ & $0(0.0)$ & $11(36.7)$ & $12(40.0)$ \\
\hline \multirow{3}{*}{\multicolumn{2}{|c|}{ Total (\%) }} & $7(23.3)$ & $11(36.7)$ & $12(40.0)$ & $30(100.0)$ \\
\hline & & & & Kappa value $=.846$ & \\
\hline & & & & $p$ value $=<0.001$ & \\
\hline \multicolumn{6}{|c|}{ Contingency table for CRQ Fair } \\
\hline \multirow[t]{3}{*}{ Obs.1 } & 1st TEST BITE (\%) & $9(30.0)$ & $0(0.0)$ & $2(6.7)$ & $11(36.7)$ \\
\hline & 2nd TEST BITE (\%) & $1(3.3)$ & $8(26.7)$ & $1(3.3)$ & $10(33.3)$ \\
\hline & 3rd TEST BITE (\%) & $0(0.0)$ & $2(6.7)$ & $7(23.3)$ & $9(30.0)$ \\
\hline \multirow[t]{3}{*}{ Total (\%) } & & $10(33.3)$ & $10(33.3)$ & $10(33.3)$ & $30(100.0)$ \\
\hline & & & & Kappa value $=.700$ & \\
\hline & & & & $p$ value $=<0.001$ & \\
\hline \multicolumn{6}{|c|}{ Contingency table for $C R Q$ Worse } \\
\hline \multirow[t]{3}{*}{ Obs.1 } & 1st TEST BITE (\%) & $11(36.7)$ & $1(3.3)$ & $0(0.0)$ & $12(40.0)$ \\
\hline & 2nd TEST BITE (\%) & $0(0.0)$ & $8(26.7)$ & $1(3.3)$ & $9(30.0)$ \\
\hline & 3rd TEST BITE (\%) & $2(6.7)$ & $0(0.0)$ & $7(23.3)$ & $9(30.0)$ \\
\hline \multirow[t]{3}{*}{ Total (\%) } & & $13(43.3)$ & $9(30.0)$ & $8(26.7)$ & $30(100.0)$ \\
\hline & & & & & Kappa value $=.797$ \\
\hline & & & & & $p$ value $=<0.001$ \\
\hline
\end{tabular}

demonstrate that a first (and only) test bite may be masking slightest distortions that, at the time of the comparative analysis, may increase the chances of producing false positives or false negatives. Although registering the suspect's incisal edges appears to be a simple protocol, problems can arise when introduced as evidence. Karazulas (1984) reported a case wherein test bites performed by the defense showed a different dental pattern different than the test bites produced by prosecution. The defendant was finally acquitted of the murder, but spent 7 years in prison and endured three trials before being proven innocent and released. Performing at least three test bites would not cause any delays or increased costs (most of the recommended materials are very economical and easy to manipulate), but would allow evaluation and selection of the highest quality sample (according to our results, any of the three registers may be the best representation of the subject's bite), as well as help detect dynamic patterns of the bite. The latter statement is currently hypothetical -at least in the forensic discipline- but it is extremely attractive to start future lines of research.

The neuromuscular engram controls a complex spatial motion pattern and although this reflex primarily affects both masticatory muscles and occlusal-muscular relationships, the engram has received so little scrutiny (Lerman 2011). The intermaxillary functional relationship should be considered not by "dogmas" of static positions but in a dynamic three-dimensional framework, that is, through relative positions of teeth, mandible and maxilla in context with oral functions and appearance (Jokstad 2012). The usual technique for obtaining test bites is to take a record with the patient's mouth passively closed based on centric relation (CR) (maximal intercuspation); this is performed on the assumption that the subject has a normal baseline psychoneuromuscular control, therefore, reproducible in time (ie, without habitual or conscious posturing) (Agbaje et al. 2013). However, number of residual teeth, occlusal support, maximum bite force, periodontal disease, difference in mandibular movement during mastication, changes in body posture, and even substrate consistency have all been described as factors that can affect neuromuscular control of chewing, muscle activity, masticatory performance (van der Bilt 2011; Kosaka et al. 2016; Sakaguchi et al. 2007) and, therefore, the final representation of the bite action: the bite mark.

Beyond these pathophysiological considerations of biting, there are other inherent limitations to the 
recording technique. Assuming that the operator has the necessary skills to perform quality test bites -another important factor considered by Yamashita et al. (2003)-, the success of tests can be conditioned by the material used and the type of bite mark. An ideal intermaxillary record material should be easy to manipulate, allow accurate records, and possess limited resistance before setting (to avoid dental or mandibular displacements during closure), then become rigid and with minimal dimensional changes after setting, even during storage (Sweeney et al. 2015; Ghazal et al. 2008). Considering that practically all materials recommended by the literature have demonstrated limitations to comply with all these aspects (Dailey 2011; Dailey et al. 2013; Ghazal et al. 2008), focus should be placed on taking special care throughout the technical procedure (Dailey et al. 2013). Although we only performed type 3 test bites (as typical bites found in cheese) -following the Webster's classification (1982)-, the goal of the criterion defined by this author fell on the type of dental features that could be registered. The wax used in this research, although not certified by the ADA (some specific products are difficult to access in our country), met the standards required for testing. We firmly believe that performing at least three records for each individual will allow detection of vulnerabilities and selection of the most appropriate registry. It is evident that this technique is not extrapolated to other types of bite marks (two-dimensional or bite marks in skin). Likewise, in the future, this could revise current protocols of comparative analysis by reformulating, for these specific cases, a new strategy of comparison "between negatives", which implies comparison of the bite marks to investigate the bite mark obtained from the suspect, and thereby seeking to replicate the bite (basically, the same principle of analysis of fingerprints). The classical methods suggest comparing negative representations of the biting teeth (the bite mark), with positive models of the suspect's teeth, both in physical or digital comparisons and overlays (Dailey 2011). Would evaluation and comparison of negatives allow morphological detection and dynamic patterns, in order to identify the perpetrator? Again, perhaps new research along these lines will answer this question.

Bite mark comparison is often used in criminal prosecutions; however, the bite mark testimony continues to be criticized for lacking scientific studies that support this type of assessment (Committee 2009). Both the uniqueness of the human dentition and the possibility of the dentition transferring to the bitten substrate (with all possible distortion possibilities) are still basic problems inherent in bite mark analysis and interpretation (Committee 2009; Saks et al. 2016). Page et al. (2011) stated that "uniqueness is impossible to prove" and added "(mistakes and misidentifications) are made because of guesswork, poor performance, lack of standards, bias and observer error". The dental information recorded on the bitten substrate should be compared to the dentition of the suspect, and although the uniqueness (the most basic concept supporting the bite mark analysis), sample selection or the applied imaging techniques still remain without scientific sufficiency (Franco et al. 2017), most current guidelines persist in considering the act of biting as only a mechanical bearing connecting two solid objects (jaws and their teeth), opening and closing with a simple hinge mechanism (American Board of Forensic Odontology 2017; Dailey et al. 2013). Although proven useful in certain experimental models, different devices designed to mimic the action of a human bite (Avon and Wood 2005; Chinni et al. 2013) are unlikely to reproduce the extreme complexity of the bite. The biomechanics of the biting action comprise not only the dental action but also the muscular relationships, occlusion, intentionality of aggression and the type of bitten material (Franco et al. 2017). Further technical attention should be given to the important phase of recording the suspect's bite pattern. Something as easy as making three records (and not just one) could significantly help control these variables and thereby improve standards of bite mark evidence.

Although the authors could be expected to perform a microscopic evaluation of the test bites (or even the use of other technological tools of greater reliability), which would have increased the range of differences to give a better understanding of the distortions/differences possible in an experiment of this nature, the purpose of this research is to optimize/improve the current ABFO recommendations for collecting evidence of suspected dentition, which do not specify a necessary minimum number of test bites to be taken (accepting by default the possibility that it is only one) nor the way in which they should be evaluated (accepting by default the possibility of being evaluated only macroscopically). From the same point of view, our research is not intended to define the concept of "slightest distortions" when precisely they have not been studied under magnification (logically in the strict sense of the word). However, we consider that this first recognition of these small patterns precisely highlights the need to use better methodologies than to only evaluate them macroscopically. The use of a DMC comparison microscope (used for analyzing firearm barrels and bullets) in bite mark analysis has already been reported as an excellent tool to enhance the ability of the forensic odontologists to present evidence correctly (Rivera-Mendoza et al. 2017; Bernitz and Kloppers 2002). In the same way, and although this experimental model can only be evaluated on a special type of bite and substrate, the high values of interrater agreement would suggest the use of similar 
models of comparison between negatives (bite marks), but adapted to other substrates bitten.

Forensic odontology, "the application of the science of dentistry to the field of law", as defined by the U.S. National Academy of Sciences (Committee 2009) represents the point of contact of two radically different scenarios. Although from a scientific point of view it seems logical to think that more testing is better then less, extrapolate this to criminal proceedings when the universally accepted recommendations of ABFO do not make it explicit. Likewise, the controversy that mires bite mark analysis can be explained -at least in part- by the indifference that seems to exist in oral science, in scientific societies, and even in applied basic research. Although the minimalist "hinge" concept of the temporomandibular joint has been overcome decades ago in the world of oral physiology, it apparently still persists in certain forensic contexts, even determining the innocence or guilt of a defendant.

\section{Conclusion}

There is no significant evidence that a first and only test bite is the highest quality test bite. This conclusion can also be applied to second or third registers, so it is recommended to take more than a single bite test in controlled situations and carry out their controls in an exhaustive way to be able to identify slightest distortions and select the one with the best conformation. Likewise, such slightest distortions would allow the dynamic interpretation of the bite action, which opens up a potential field of research in the bite mark analysis.

\section{Abbreviations \\ ABFO: American Board of Forensic Odontology; ADA: American Dental Association; CEC: [Comité de Ética Científico] Ethical Scientific Committee of the Universidad de La Frontera; CR: Centric Relation; CRQ: Categories of Record Quality; FRM: Fernando Rivera-Mendoza (author 1); GMF: Gabriel M. Fonseca (author 4)}

\section{Acknowledgements}

Not applicable

\section{Funding}

The authors declare that there is no funding for the research reported.

\section{Availability of data and materials}

Not applicable

\section{Authors' contributions}

The individual contributions of authors to the manuscript is specified below: RMF: Study concepts, study design, data acquisition, data analysis and interpretation, manuscript editing. MdIHS: Study concepts, data analysis and interpretation, manuscript editing, manuscript review. N-CP: Study design, quality control of data and algorithms, data analysis and interpretation, statistical analysis. FGM: Study concepts, study design, data analysis and interpretation, manuscript preparation, manuscript editing, manuscript review. All authors read and approved the final manuscript.

\section{Ethics approval and consent to participate}

The present study was approved by the Ethical Scientific Committee of the Universidad de La Frontera (CEC) by act no. 079/2015. The CEC is an agency accredited by the Regional Ministerial Health Secretariat of Chile. The experiments carried out in this study were performed within the guidelines of the CEC for human studies and were carried out under the strict supervision of a registered dental practitioner (FRM). Also, all the subjects have been fully informed of the experimental procedure and consent was obtained prior to the experiment. Written informed consent for publication was obtained from the person shown in Fig. 1c.

\section{Consent for publication}

Not applicable

\section{Competing interests}

The authors declare that they have no competing interests.

\section{Publisher's Note}

Springer Nature remains neutral with regard to jurisdictional claims in published maps and institutional affiliations.

\section{Author details}

'Programa de Magister en Odontología, Facultad de Odontología, Universidad de La Frontera, Temuco, Chile. ${ }^{2}$ Forensic Dentistry Lab, Centro de Investigación en Odontología Legal y Forense (ClO), Facultad de Odontología, Universidad de La Frontera, Francisco Salazar 01145, Building L, 4811230 Temuco, Chile. ${ }^{3}$ Department of Forensic Medicine and Forensic Odontology, University of Granada, Granada, Spain. ${ }^{4}$ Centro de Investigación en Ciencias Odontológicas (CICO), Facultad de Odontología, Universidad de La Frontera, Temuco, Chile.

Received: 13 October 2017 Accepted: 22 February 2018

Published online: 02 March 2018

\section{References}

Agbaje JO, Sun Y, Lambrichts I, Politis C (2013) Problems during orthognathic surgery resulting from errors in diagnostic wax bite. J Craniofac Surg 24(3): 999-1001.

American Board of Forensic Odontology. ABFO Reference Manual (Updated April 2017) http://abfo.org/wp-content/uploads/2012/08/ABFO-Reference-ManualApril-2017-v7.pdf. Accessed 22 Jul 2017.

Avon SL, Wood RE (2005) Porcine skin as an in-vivo model for ageing of human bite marks. J Forensic Odontostomatol 23(2):30-39.

Bernitz H, Kloppers BA (2002) Comparison microscope identification of a cheese bitemark: a case report. J Forensic Odontostomatol 20(1):13-16.

Chinni SS, Al-Ibrahim A, Forgie AH (2013) A simple safe, reliable and reproducible mechanism for producing experimental bite marks. J Forensic Odontostomatol 31(1):22-29.

Committee on Identifying the Needs of the Forensic Sciences Community, National Research Council (2009) Strengthening forensic science in the United States: a path forward. National Academies Press, Washington, pp 173-176.

Dailey JC (2011) Methods of comparison. In: Dorion RBJ (ed) Bitemark evidence. A color atlas and text, 2nd edn. CRC Press, Boca Raton, pp 469-489.

Dailey JC, Golden GS, Senn DR, Wright FD (2013) Bitemarks. In: Senn DR, Weems RA (eds) ASFO manual of forensic Odontology, 5th edn. CRC Press, Boca Raton, pp 257-324.

Franco A, Willems G, Souza P, Coucke W, Thevissen P (2017) Uniqueness of the anterior dentition three-dimensionally assessed for forensic bitemark analysis. J Forensic Legal Med 46:58-65.

Ghazal M, Albashaireh ZS, Kern M (2008) The ability of different materials to reproduce accurate records of interocclusal relationships in the vertical dimension. J Oral Rehabil 35(11):816-820.

Johnson LT (2011) The suspect. In: Dorion RBJ (ed) Bitemark evidence. A color atlas and text, 2nd edn. CRC Press, Boca Raton, p 465.

Jokstad A (2012) Methodological challenges in the study of dental occlusion. J Oral Rehabil 39(7):480-488.

Karazulas CP (1984) The presentation of bite mark evidence resulting in the acquittal of a man after serving seven years in prison for murder. J Forensic Sci 29(1):355-358.

Kosaka T, Ono T, Kida M et al (2016) A multifactorial model of masticatory performance: the Suita study. J Oral Rehabil 43(5):340-347.

Lerman MD (2011) The muscle engram: the reflex that limits conventional occlusal treatment. Cranio 29(4):297-303.

Little RM (1975) The irregularity index: a quantitative score of mandibular anterior alignment. Am J Orthod 68(5):554-563. 
Page M, Taylor J, Blenkin M (2011) Uniqueness in the forensic identification sciences-fact or fiction? Forensic Sci Int 206(1-3):12-18.

Rivera-Mendoza F, Martín-de-Las-Heras S, Navarro-Cáceres P, Fonseca GM (2017) Bite mark analysis in foodstuffs and inanimate objects and the underlying proofs for validity and judicial acceptance. J Forensic Sci. https://doi.org/10. 1111/1556-4029.13586.

Sakaguchi K, Mehta NR, Abdallah EF et al (2007) Examination of the relationship between mandibular position and body posture. Cranio 25(4):237-249.

Saks MJ, Albright T, Bohan TL et al (2016) Forensic bitemark identification: weak foundations, exaggerated claims. J Law Biosci 3:538-575.

Scott J (2010) Accurate bite records: improving restorative predictability. Dent Today 29(1):124 126-127.

Sheasby DR, MacDonald DG (2001) A forensic classification of distortion in human bite marks. Forensic Sci Int 122(1):75-78

Sweeney S, Smith DK, Messersmith M (2015) Comparison of 5 types of interocclusal recording materials on the accuracy of articulation of digital models. Am J Orthod Dentofac Orthop 148(2):245-252.

The Dawson Academy Blog (2008) The bite record directive: the need to properly record centric relation. http://thedawsonacademy.typepad.com/ alumni/2008/11/the-bite-record-directive-the-need-to-properly-record-centricrelation-1.html. Accessed 22 Jul 2017.

van der Bilt A (2011) Assessment of mastication with implications for oral rehabilitation: a review. J Oral Rehabil 38(10):754-780.

Webster G (1982) A suggested classification of bite marks in foodstuffs in forensic dental analysis. Forensic Sci Int 20(1):45-52.

Yamashita S, Igarashi Y, Ai M (2003) Tooth contacts at the mandibular retruded position, influence of operator's skill on bite registration. J Oral Rehabil 30(3): $318-323$.

\section{Submit your manuscript to a SpringerOpen ${ }^{\circ}$ journal and benefit from:}

- Convenient online submission

- Rigorous peer review

- Open access: articles freely available online

- High visibility within the field

- Retaining the copyright to your article

Submit your next manuscript at $\boldsymbol{s p r i n g e r o p e n . c o m ~}$ 